Mean Places of the Comparison Stars for r888.o.

\begin{tabular}{|c|c|c|c|c|c|c|c|}
\hline a $\times 888.0$ & $\delta \times 888.0$ & Authority & $*$ & Mg. & $a \mathrm{I} 888.0$ & $\delta^{\prime}$ I 888.0 & Authority \\
\hline $40^{m} 40^{s} \cdot 70$ & $+39^{\circ} 43^{\prime} 4^{\prime \prime} .7$ & BB.VI + $39^{\circ} .2559$ & 5 & 8.5 & I $5^{\mathrm{h}} \quad 6^{\mathrm{m}} \quad 9^{\mathrm{s}} \cdot 34$ & $+19^{\circ} \mathbf{1}^{\prime} 5^{2 \prime \prime} 7$ & $W_{2} \times 5: 85$ \\
\hline 7.45 & $+3825 \quad 20.5$ & $W_{2} \times 2$ h 1046 & 6 & $9 . \mathrm{I}$ & I5 I6 33.42 & +1728 & $6 \mathrm{cp}$. with $\mathrm{W}_{2} \times 5^{\mathrm{h}} \cdot 4 \times 5$ \\
\hline $3^{8} \quad 4^{6.43}$ & $+3334 \quad 40.4$ & $\mathrm{~W}_{2} \mathrm{I} 3^{\mathrm{b}} 78 \mathrm{I}$ & 7 & 9.2 & I5 $56 \quad 59.47$ & $+955 \times 3.0$ & BB.VI 2943 \\
\hline 0.94 & +325445.6 & ro cp. with $\mathrm{W}_{2}$ I $3^{\text {h }} \cdot 93^{2}$ & 8 & 6.4 & 4.23 & $+8495^{6 . I}$ & $W_{1} I 6^{h} \cdot 1$ \\
\hline
\end{tabular}

Notes.

ung. 29. Comet rather low and faint. - Sept. 5. Comet rather low and faint. - Sept. 24. Stopped by fog $\left({ }^{3} / 3\right.$ wt. $)$. Oct. 8 . Comet very faint. Obs. difficult. - Oct. ıo. Comet very faint. Obs. rather difficult:

\title{
Observations de la Comète 1888 III (Brooks Août 7)
}

à l'Observatoire royal de Bruxelles, à l'équat. de l’Est (om5), par E. Sturyaert, astr. adj., communiquées par le Directeur $F$. Folie. Micromètre circulaire.

\begin{tabular}{|c|c|c|c|c|c|c|c|c|c|}
\hline T. M. Brux. & $\Delta a$ & $\Delta \delta$ & $\mathrm{Cp}$. & $a$ app. & $\log p . A$ & $\delta$ app. & $\log p \cdot A$ & Red. ad 1. app. & * \\
\hline $10^{\mathrm{h}} 25^{\mathrm{m}} 5^{2^{\mathrm{s}}}$ & $+4^{\mathrm{m}} 6^{\mathrm{s}} \mathrm{I} 3$ & $+5^{\prime} 4^{\prime \prime} 7$ & 6 & $10^{\mathrm{h}} 29^{\mathrm{m}} 54^{\mathrm{s}} \cdot 9^{6}$ & .543 & $+44^{\circ} 5^{\circ} 3$ & $.89^{6}$ & $-0.78+1.2$ & 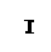 \\
\hline 10 4437 & -217.90 & +7448 & 4 & I0 3742.58 & & +444933.4 & 05 & $-0.77+\mathrm{r} .9$ & 2 \\
\hline Io 3343 & +2 I 5.10 & +96.3 & 6 & I0 $53 \quad 10.08$ & & $+444 \mathrm{I}$ I 7.2 & 94 & $-0.76+2.2$ & 3 \\
\hline I0 3343 & - I 14.93 & +10 22.8 & 6 & 10 5310.03 & & +4441 I 3.0 & 394 & $-0.75+$ & 4 \\
\hline $9393 \mathbf{I}$ & -223 & +053.7 & 2 & - & & - & $5 \circ$ & $-0.74+$ & 5 \\
\hline 9.3931 & -324.40 & +219 & 2 & $\circ 4^{2}$ & & $+44335^{8.7}$ & 50 & $-0.74+2.8$ & 6 \\
\hline ro 3310 & -o 37.80 & +519.1 & $=$ & 一 & & 一 & 82 & $58+3.9$ & 7 \\
\hline $\begin{array}{lll}8 & 53 & 44\end{array}$ & +29.65 & +49.7 & 5 & I 2323 I & & +402222.6 & 73 & $-0.50+$ & 8 \\
\hline $8 \quad 5344$ & +14.00 & $-\circ 3^{8.2}$ & 5 & - & & - & 73 & $50-1$ & 9 \\
\hline 9 I 59 & to 6.95 & -619.9 & 5 & I 3949,80 & & $+394^{6} 54.1$ & & $47+$ & Io \\
\hline 9196 & -218.90 & $-3 \quad 54.4$ & 8 & $1253 \quad 47.84$ & & $+3^{8} 35$ I 3.7 & 98 & $41+6.9$ & I I \\
\hline $934 \quad 53$ & $+33^{I}$ & +02.1 & 3 & 13039.09 & & +3757 & 3 & $40-+$ & $\mathrm{r}_{2}$ \\
\hline $\begin{array}{lll}9 & 34 & 53\end{array}$ & to 54.68 & +414.6 & 5 & $13 \bigcirc 39.02$ & & +375653.4 & I 3 & $39+$ & I 3 \\
\hline 93453 & -031.80 & +53 I.I & 5 & - $39.7 \mathrm{I}$ & & +375658.1 & I 3 & $38+7.0$ & I 4 \\
\hline $934 \quad 53$ & -I 24.20 & +454.0 & 3 & 039.62 & & +375657.8 & I 3 & $-0.38+7.1$ & r 5 \\
\hline 9415 & $-1 \times 5.16$ & $\therefore 10 \quad 39.9$ & 9 & 7 I 2.15 & 9.668 & +37 I $8 \times 5 . x$ & 0.782 & $-0.3^{6}+7.3$ & I 6 \\
\hline
\end{tabular}

Positions moyennes des étoiles de comparaison pour I888.0.

\begin{tabular}{|c|c|c|c|c|c|c|}
\hline 888.0 & $\delta_{1} 888.0$ & Autorité & $*$ & $\alpha \mathrm{I} 888.0$ & $\delta_{I} 888.0$ & Autorité \\
\hline $5^{\mathrm{m}} 49^{\mathrm{s}} \cdot 6 \mathrm{I}$ & $+44^{\circ} 45^{\prime} 3^{\prime \prime \prime} 3$ & $W_{2} 10^{h} \cdot 470$ & 9 & ${ }^{1} 2^{\mathrm{h}} 3 \mathrm{I}^{\mathrm{m}} 26^{\mathrm{s}}$ & $+40^{\circ}=3^{\prime}$ & DM. +40.2554 \\
\hline 1.25 & +444146.7 & Lal. 20726 & ro & I $23943 \cdot 3^{2}$ & $+3953 \quad 7.7$ & $W_{2} 12^{\mathrm{h}} \cdot 795-6$ \\
\hline $55 \cdot 74$ & $+443^{2} 8.7$ & $W_{2} 10^{h} 988$ & I I & I $2 \quad 5^{6} \quad 7.15$ & $+3^{8} 39$ & Lal. $24246-7$ \\
\hline 25.73 & $+4430 \quad 47.8$ & $W_{2}$ Iot. $105 \mathrm{I}$ & I 2 & I $257 \quad 7.96$ & +375659.4 & $W_{2} I_{2} I_{1} O_{3}$ \\
\hline 6 & +4433 & DM. $+44^{\circ} 2059$ & I3 & I 25944.73 & $+375^{2} 3 \times .9$ & $W_{2}$ I $2^{\mathrm{h}} \times 145$ \\
\hline 7.17 & $+443^{1} 3^{6.9}$ & $W_{2} I^{\mathrm{b}}{ }^{\mathrm{I}} 3-\mathrm{I} 4$ & 14 & $\times 3 \quad 1 \times .89$ & +375120.0 & $W_{2} \times 2 ! 1173$ \\
\hline 0 & +4337 & DM. $+43^{\circ}: 2 \times 34$ & 15 & 1324.20 & +375156.7 & $W_{2}$ 1 ${ }^{\mathrm{h}} \times 187$ \\
\hline 22.15 & $+40 \times 8 \quad 7.0$ & $W_{2} \times 2^{h} \cdot 6$ I 3 & I6 & I3 827.67 & +372847.7 & Lal, 24579 \\
\hline
\end{tabular}

eobachtung des Faye'schen Cometen $1888 \mathrm{IV}$ auf der Sternwarte in Wien.

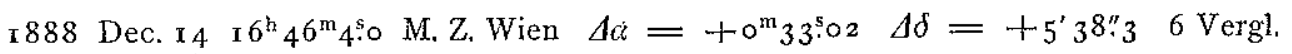

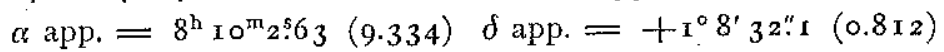

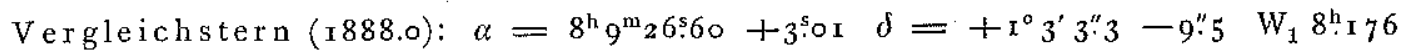

omet sehr schwach; er besitzt einen schwer merkbaren Kern, der von einer runden, gleichmässig hellen Coma 40" Durchmesser umgeben ist.

Vähring 1888 Dec. $x 6$.

R. Spitaler. 